\title{
Investigation of dynamics of roll forming processes
}

\author{
Leonid A. Kondratenko ${ }^{1}$, and Lubov I. Mironova ${ }^{2, *}$ \\ ${ }^{1}$ State Science Center RF of TsNIITMash, Moscow, Russian Federation \\ ${ }^{2}$ National Research Nuclear University MEPhI (Moscow Engineering Physics Institute) Moscow, \\ Russian Federation
}

\begin{abstract}
This article contains the analysis of tube expander dynamics in complex interaction of structural elements of heat-exchange tubes attachment assembly in the process of roll-forming operation, description of dynamic process theoretical aspect. It is shown that torque variations lead to velocity fluctuations and influence the service life of operative parts of tube expander and quality of tube attachment assemblies.
\end{abstract}

Modern power, oil-processing and chemical industries make full use of apparatuses where the attachment of heat-exchange tubes is carried out by flaring their ends with the help of rolling.

Despite considerable (almost eternal) accumulated experience with the use of tube expanders, in many cases any change in the parameters of tubes or rolling tools leads to unexpected negative results - inadmissible peeling of the tube inner surface, joint failure, rapid tool wear, etc. Moreover, the indicators signaling a violation of tube attachment quality often manifest themselves during the installation of a heat exchanger at the site of use and even during its operation. This indicates a lack of study of this process and requires new approaches and methods for studying dynamic elastoplastic processes in the technological influence zones.

At present, production of heat-exchange energy equipment is based on the normative documentation developed in the previous century. It is based on the methodology of earlier studies with large assumptions [1-3]. In their basis, the roll forming process is considered as a gradual alignment of two almost concentric tube surfaces and a hole in a tube sheet with a subsequent pressing. This approach is correct for the processes of hydraulic or press rolling. In the case of tube expander rolling, we should indicate some features that do not fully coincide with this point of view and require additional studies of such processes as:

- plastic deformation of the tube by conical tube expanders whose rotation axis does not match the tube axis;

- contact interworking of conical tube expanders and the surface of the tube being deformed;

- dynamics of the working part of the tube expanders, especially with a long tool length and instability of its position, as well as in cases of using different types of drives.

\footnotetext{
*Corresponding author: mironova 1ub@mail.ru
} 
The rolling process is usually considered from the standpoint of a static process. The contact of the tube expanders with the tube in this case can be analyzed using the solutions obtained in [4-7].

In reality, fluctuations with different amplitude and frequency take place here, affecting both the tool operability and the tube attachment quality when manufacturing and repairing heat exchangers [8].

The study of dynamic peculiarities of this attachment technology is of scientific and practical interest.

In tube expander rolling, tube deformation is due to the tube bending at its contact points with the tube expanders and subsequent plastic changes of dimensions. Therefore, the rolling process should be considered taking into account the multifarious interaction of the contacting surfaces at each loading point in combination with the contact problem. Fig. 1 shows the scheme of rolling.

A tight bonding of the tube with the surface of the tube grid hole is ensured by the torque of the spindle (3) and by pressing of the tube expander (1) in some vicinity of the contact point of the tube (8) and the tube expander.

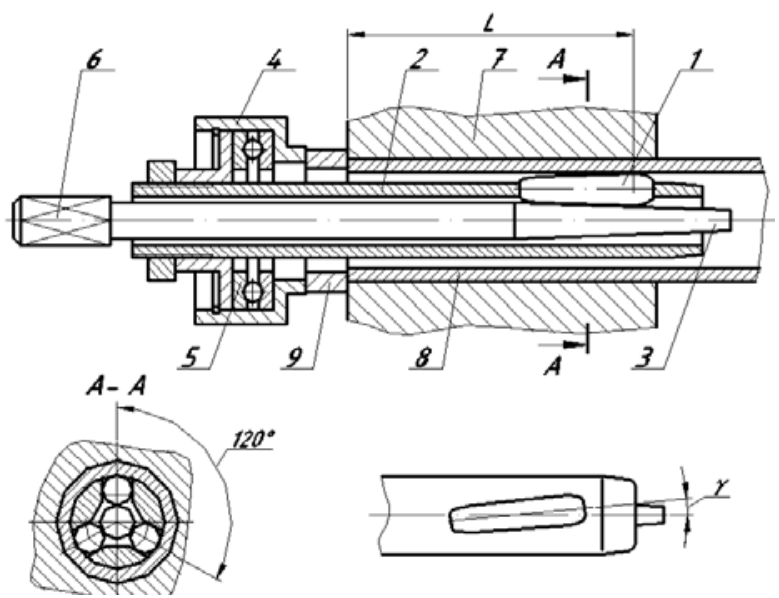

Fig. 1. Scheme of rolling the tube attachment assembly into the tube grid: $1-$ tube expander, $2-$ body, 3 - spindle, 4 - holder, 5 - rolling bearing, 6 - extension tube, 7 - tube sheet, tube.

In the tube expander coverage area, the tube metal becomes plastic when some of the layers move along the tube expander surface trajectory, and some of them are stretched and compressed, pressing against the wall of the hole [8].

Note that body (2), where the tube expanders are placed, rotates in the same direction with the spindle, but with a different frequency.

The length of the rolling body is chosen based on the design of the tube attachment assembly in the tube grid and the length of the rolling section. Its dimensions can reach large values. In this case, the moment of inertia of the body increases as it rotates, which under certain conditions can have a negative effect on the rolling process [8,9]. Let us analyze the dynamics of the rolling process.

Let us assume that the rolling shown in Fig. 1 is used for tube attachment. The rolling process is described in detail in [8-13]. Its peculiarity lies in the fact that the rotating spindle is retracted and with the conical part through the tube expanders plastically deforms the tube. At the instant of the first contact of the tube section outer surface and the inner surface of the tube sheet hole, the contact is made at three points. From this moment on, the tube deformation conditions change significantly, especially after the moment when the contact does not stop at the indicated three points when the tube expander moves to another 
place.

If, prior to the first contact, a segment of approximately $1 / 3$ of the tube circumference was stretched between a pair of tube expanders, then each tube expander acts on a section of the tube located between two closer points. Moreover, as the spindle retracts, the length of such section can reduce intermittently [8]. We assume that the cylindrical shell is subjected to the action of forces distributed symmetrically about the cylinder axis.

To study the dynamic characteristics of the process, we use the method described in [13, 14]. As a computational model, we take a generalized model of an inertial disk rotating at the end of rod [14]. We denote the angular velocities of rotation of the spindle cross sections at the liner and near the working part, as $\Omega_{1}, \Omega_{2}$, respectively. Neglecting the relaxation phenomena, the rolling system dynamics is described by the following differential equations

$$
\begin{gathered}
\tau(t) W_{p}=M_{\kappa p 0}(t)+M_{m p}(t)+J_{n p} d \Omega_{2} / d t ; \\
\vartheta d \tau / d t=\Omega_{1}-\Omega_{2},
\end{gathered}
$$

where $\tau$ is the maximum tangential stresses in the spindle section; $W_{p}$ is the polar moment of the spindle section resistance at the liner boundary; $M_{\kappa p 0}$ is the torque ensuring deformation of the tube; $M_{m p}$ is the friction torque loss; $J_{n p}$ is the moment of inertia of the tool rotating parts, reduced to the spindle axis; $t$ the time of rolling, $\vartheta$ is the coefficient that characterizes the torsional elasticity of the spindle.

From the solution of system (1) after the corresponding transformations when $\Delta \Omega=\Omega_{1}$ $\Omega_{2}$, we arrive at the following equation of motion

$$
W_{p} \vartheta^{-1} \Delta \Omega(t)+d \Delta \Omega / d t+J_{n p} d^{2} \Delta \Omega / d t^{2}=-M_{k p 0}(t),
$$

which, in fact, is the known equation of forced fluctuations [13].

The relationship between the maximum stresses in the rod, the torque and the angular velocity of motion can be described by correlation $[11,12]$

$\tau=\left[M_{\kappa p 0}(t)+h_{k} \Omega_{2}(t)\left(1+T_{н к} p\right)\right] W_{p}^{-1}$.

Here $h_{k}$ is the friction loss coefficient; $T_{н к}$ is the time constant $T_{н \kappa}=J_{n p} / h_{k}$,

Thus, the dynamics of the fluctuation system of the rolling tool depends on many parameters, including: spindle torque, geometric characteristics of the body, spindle and tube expander, the length of rolling and the rolling equipment parameters. Let us consider each of them in detail.

During rolling, the tube expander, rotating at frequency $\Omega_{p}$, is pressed against the tube by a spindle rotating about its own axis with frequency $\Omega$. At the same time as the spindle deepens into the tube, the force interaction between the tube expander and the spindle increases. The maximum force is $F_{r c i}$, Fig. 2. Thus, when setting up one attachment assembly in a single-roll cycle, torque $M_{\kappa p}$ acting on the spindle, varies from a minimum to a maximum [8]. The theoretical definition of the torque, which ensures plastic deformation of the tube and a dense bond, should be considered from the kinematics of the force interaction at the point of contact of the tube expander and then with its subsequent indenting into the inner surface of the tube, Fig. 2.

Frequency of the tube expander rotation relative to the shell $n_{p \kappa}$ is bound by the formula

$$
n_{p \kappa}=0,75 n_{6} \cos \alpha_{6 \kappa},
$$

where $n_{b}$ is the spindle rotation frequency. 


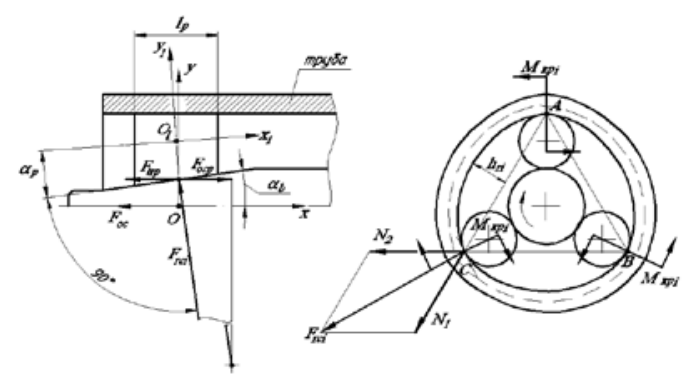

Fig. 2. Design scheme of the force interaction of a tube expander, a spindle and a tube.

The given inertia moment of the system can be defined as the sum of the inertia moment of the rotating body $J_{\kappa}$ of the tube expander and the moment of transfer pair $J_{\phi p}$ "spindle tube expander". The proper inertia moment of the tube expander is neglected. Then

$$
\begin{gathered}
J_{\kappa}=0,5 \gamma_{\mu \kappa} g^{-1} l_{\kappa} \pi\left(R_{н \kappa}^{4}-r^{4}{ }_{в \kappa}\right) K_{1} \\
J_{B p}=0,5 z \pi l_{p} \gamma_{M p}\left[i_{p} r_{p c}^{2}+K_{l}\left(r_{p c}+r_{B c}\right)^{2}\right] r_{p c}^{2} / g .
\end{gathered}
$$

Here $\gamma_{M \kappa}$ is specific weight of the metal shell; $l_{\kappa}$ is the outer, inner diameters and body length; $R_{H \kappa}, r_{\varepsilon \kappa}, K_{1}$ is the gear ratio between the tube expander and the shell; $r_{p c}, r_{B c}$ are the average diameters of the tube expander and the spindle, respectively; $i_{p}$ is the gear ratio between the tube expander and the spindle.

Let us assume that between the tube expander and the tube inner surface, the normal specific force $F_{q l}$ is constant over the entire length of the contact. As a result of interaction of the elementary section of the surface of the tube expander $d x$ long and the tube in a plane perpendicular to the spindle rotation axis, an elementary circumferential force $F_{t r}$ develops at the point of their contact, and it can be determined as

$$
d F_{t r}=d M_{\kappa p 0} / r_{p i}
$$

It should be noted that the tube expander section radius is a variable value along the spindle length and can be determined by the function of the average radius of the section $r_{p c}$, if the coordinate axis $\mathrm{Y}_{1} \mathrm{O}_{1} \mathrm{X}_{1}$ is placed in the center of the tube expander. In this case, the radius is determined by the relation

$$
r_{p i}=r_{p c}\left(1+\operatorname{tg} \alpha_{p} \cdot x / r_{p c}\right)=k r_{p c} .
$$

The tube expander angle relative to the spindle has a small value $(\operatorname{tg} \alpha=1 / 120 \div 1 / 180)$. We assume that coefficient $k$ equals one. Taking into account (3) and (4), let us put down the torque developing on the tube expander

$$
M_{\kappa p 0}=\int_{-l / 2}^{+l / 2} r_{p c} \cdot F_{q l} \boldsymbol{\mu}_{p m} d x=\boldsymbol{\mu}_{p m} r_{p c} F_{q l} l .
$$

Here $\mu_{p m}$ is the coefficient of friction between the tube and the tube expander; $l$ is the contact length. The circumferential force acting on the tube expander surface and perpendicular to $\mathrm{OX}$ is

$$
F_{t r}=\mu_{p m} l F_{q l} \cos \gamma .
$$

The force acting perpendicular to the tube expander axis is determined by the formula 


$$
F_{1}=F_{t r} / \cos \gamma
$$

The tube expanders in the process of hypocycloidal rolling of the tube inner surface push it in the opposite direction with a force

$$
F_{o c l}=F_{t r} \operatorname{tg} \gamma .
$$

Assuming that the tube expander fully adheres to the spindle surface and the specific normal load to the generatrix between the tube expander and the spindle $F_{q 2}$ is constant along the contact length, we write down the following correlations:

- the torque generated by the working (conical) part of the spindle

$$
M_{\kappa p s i}=F_{q 2} \mu_{p в} r_{\varepsilon c i} ;
$$

- power used for tube expander operation

$$
N=F_{q 2} \mu_{p s} r_{s c i} \Omega .
$$

Here $r_{c c i}$ is the average current radius of the spindle working part, changing as the spindle deepens into the tube.

Thus, all the force factors are determined. With this in mind, we will give the final formulas for calculating the forces acting on each tube expander:

- radial force

$$
F_{r c i}=k_{c} M_{\kappa p 0} \operatorname{tg} \gamma /\left(z r_{p i} \sin \alpha_{B}\right) ;
$$

- circumferential force acting on the outside diameter

$$
F_{t}=M_{\kappa p 0} /\left(3 k_{c} r_{p c}\right)
$$

- axial force

$$
F_{o c p z}=F_{t r} \sin \gamma+F_{r c i} \mu_{p m} \sin B+F_{r c i} \mu_{p m} \cos (B+\gamma) \cos \gamma / \operatorname{tg}\left(\gamma+B+\rho_{k}\right)
$$

Here $k_{c}$ is the coefficient that takes into account unevenness of the load distribution between the tube expanders. These formulas have practical confirmation [8].

Since the forces acting in the rolling process depend on the torque and the rolling parameters, in accordance with equation (2), there are rotation frequency fluctuations of the spindle and the tube expanders, which significantly affect the quality of the tube attachment assembly.

Fig. 3 shows the experimental oscillogram of the rolling process of a tube $\varnothing 16 \times 1,4$ made of steel $08 \mathrm{X} 18 \mathrm{H} 10 \mathrm{~T}$ in a bed with a hole $\varnothing 16,4 \mathrm{~mm}$ at $M_{\kappa p}=4.4 \mathrm{Nm}, \mathrm{n}=400 \mathrm{rpm}$ by rolling. 


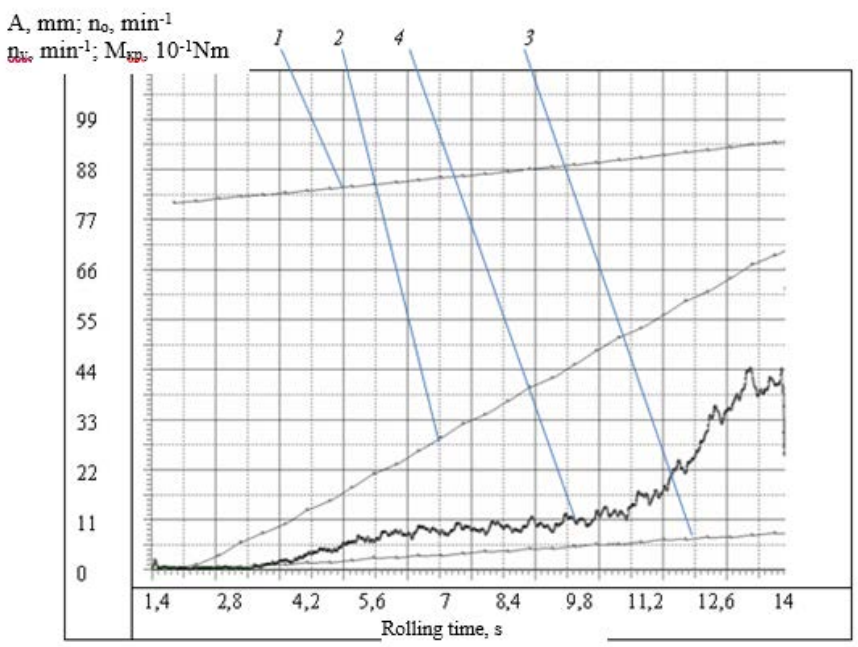

Fig. 3. Oscillogram of mechanical rolling: 1 is the axial movement of the spindle, $\mathrm{mm} ; 2$ is the number of spindle revolutions (nv), rev; 3 is the number of rolling body revolutions (no), rev; 4 is the torque at rolling $(\mathrm{Mcr}), \mathrm{dNm}$

The experiment results show that rotation of the spindle is accompanied by regular fluctuations of the torque. The number of such fluctuations is approximately equal to the number of spindle revolutions, and the amplitude after some damping comes up to $(0,15 \ldots$ $0,20) M_{k p}$. The probable cause of such fluctuations is the slip of the tube expander with respect to tube [9].

It should be noted that this amplitude of fluctuations was obtained after damping in the tensometric gauge of the test bench. Such fluctuations, especially with significant axial loads, have a significant effect on the resource of the tool and the quality of rolling.

Experimental and analytical studies show that all rolling parts are under the influence of variable forces, which, with the continuous processing of thousands of holes (for example, in the LDPE boards), affect the workability of rolling. Under certain conditions, due to fatigue stresses, the spindle, tube expanders, and shell may break down. Such an accident in a critical product manufacture is extremely undesirable, as it causes damage to the internal surfaces of the tubes and their subsequent possible corrosion damage. In addition, fluctuations in the torque lead to fluctuations in the speed of tube expanders, which affects the stressed state of the parts of the tube attachment assembly.

\section{References}

1. Tkachenko G. P., Brif V. M. Manufacturing and repair of shell-and-tube heatexchange equipment. (Machine-building, Moscow, 1980)

2. Yuzik S. I., Tube expanding in ship heat exchangers. (Shipbuilding, Leningrad, 1978)

3. Novokshchnov S. L., Mathematical modelling of roll-forming processes. Transactions of VIII All-Russian with international engagement Scientific and Technical Conference and School of Young Scientists, Postgraduate Students and Students. Voronezh. VSTU, p. 89-92. (2007)

4. Johnson K. L., Contact Mechanics (Mir, Moscow, 1989) 
5. Bulanov E. A., Universal relationship between deformation parameters and load at elastic plastic ball indentor penetration into the elastic half-space. Problems of machine-building and machine reliability. No. 1, pp. 25-29 (2009)

6. Koteneva N.V., Elastic-plastic contact of a smooth sphere with a planar surface under dynamic loading. Izv. Altai State Technical University. Vol. T308, No. 2, pp. 115-1119 (2005)

7. Nadai A. L., Plasticity (Plastichnost'. ONTI, 1936)

8. Kondratenko L. A. Roll-forming of heat-exchange tubes, Sputnik (Moscow, 2015)

9. Kondratenko L.A., Mironova L.I., To the question of hardening of material of milling connections in heatexchange devices. Engineering \& Automation Problems. No. 3, pp. $106-112$ (2016)

10. Kondratenko L.A., Mironova L.I., Features of the formation of internal surface defects of heat-exchanging tubes during their conversion, Proceedings of the Scientific Readings. I.A. Odinga "Mechanical properties of modern structural materials." Moscow, IMET RAS, pp.59-60 (2016)

11. Kondratenko L.A., Terekhov V.M., Mironova L.I., On the effect of the dynamics of the roller rolling on the quality of manufacture of heat exchangers of nuclear power units, Heavy engineering construction, No. 3, pp. 10 - 14 (2016) (In Russ.).

12. Kondratenko L., Terekhov V., Mironova L., The aspects of roll-forming process dynamics. Vibroengineering PROCEDIA. AT the 22end International Conference on Vibroegineering, Moscow, pp. 460-465 (2016)

13. Kondratenko L. A., Vibrations and speed regulation methods of movement of technological objects (Moscow, MRSU, 2005)

14. Kondratenko L.A., Terekhov V.M., Mironova L.I., About one method of research torsional vibrations of the core and his application in technologies of mechanical engineering, Engineering \& Automation Problems, No. 1, pp. 133-137 (2017)

15. Yuvorsky B. M., Detlaf A. A., Physics handbook (Science, Moscow, 1974) 